\title{
Sleep-learning impairs subsequent awake-learning
}

\author{
Simon Ruch ${ }^{*}$, , Marc Alain Züst ${ }^{1}$, Katharina Henke ${ }^{2}$ \\ Cognitive Neuroscience of Memory and Consciousness, Institute of Psychology, University of Bern, Fabrikstrasse 8, 3012 Bern, Switzerland
}

\section{A R T I C L E I N F O}

\section{Keywords:}

Slow-wave sleep

Synaptic homeostasis

Consciousness

Vocabulary acquisition

Hippocampus

\begin{abstract}
A B S T R A C T
Although we can learn new information while asleep, we usually cannot consciously remember the sleep-formed memories - presumably because learning occurred in an unconscious state. Here, we ask whether sleep-learning expedites the subsequent awake-learning of the same information. To answer this question, we reanalyzed data (Züst et al., 2019, Curr Biol) from napping participants, who learned new semantic associations between pseudowords and translation-words (guga-ship) while in slow-wave sleep. They retrieved sleep-formed associations unconsciously on an implicit memory test following awakening. Then, participants took five runs of paired-associative learning to probe carry-over effects of sleep-learning on awake-learning. Surprisingly, sleeplearning diminished awake-learning when participants learned semantic associations that were congruent to sleep-learned associations (guga-boat). Yet, learning associations that conflicted with sleep-learned associations (guga-coin) was unimpaired relative to learning new associations (resun-table; baseline). We speculate that the impeded wake-learning originated in a deficient synaptic downscaling and resulting synaptic saturation in neurons that were activated during both sleep-learning and awake-learning.
\end{abstract}

\section{Introduction}

Growing evidence suggests that we can unconsciously process and store new information during deep sleep and that this information transfers into wakefulness (Andrillon, Pressnitzer, Léger, \& Kouider, 2017; Arzi et al., 2012, 2014; Ruch, Koenig, Mathis, Roth, \& Henke, 2014; Ruch \& Henke, 2020; Züst, Ruch, Wiest, \& Henke, 2019). Following awakening, we cannot consciously remember our sleepformed memories because we formed those memories in the unconscious state of deep sleep. Sleep-formed memories merely exert implicit, indirect effects on our awake behavior (Ruch \& Henke, 2020). This may limit the usefulness of sleep-learning for conscious information processing.

Here, we explore whether sleep-learning might expedite the subsequent awake-learning of the same information that had previously been sleep-played. We figured that a first bout of carving a new memory trace during sleep might provide a basis, which ensuing awake-learning can build on. To test this hypothesis, we analyzed previously unreported data acquired in a project on vocabulary learning during slow-wave sleep (SWS) (Züst et al., 2019). These authors observed that new vocabulary was encoded and stored for the long-term during SWS, if the formation of new semantic associations between fictitious foreign-words (pseudowords) and translation-words coincided with peaks of sleep slow-waves (i.e., the positive half waves of slow-waves identified in the electroencephalogram; Fig. 1A). Because the formation of a semantic association cannot take place before the second word is being played, sleep-learning can only be effective when the second word of a pair which is the time when associative learning occurs - coincides with a brief period of increased cortical excitability and plasticity, i.e., a slowwave peak. Neuronal network properties resemble those of the waking state during a slow-wave peak (Cox, Korjoukov, de Boer, \& Talamini, 2014; Destexhe, Hughes, Rudolph, \& Crunelli, 2007; Schabus et al., 2012; Sirota \& Buzsáki, 2005). Peak-associated paired-associative learning yielded a significant associative retrieval performance on the implicit memory test given after awakening (Fig. 1B). Interestingly, sleep-learning, although unconscious, appeared to have recruited the episodic memory system. This was suggested by neuroimaging data collected during implicit memory testing that showed activity increases in the hippocampus and left hemisphere language areas during correctly versus incorrectly responded retrieval trials (Züst et al., 2019). Hence, vocabulary learning during sleep might recruit the same hippocampalneocortical network that is also involved in vocabulary learning

\footnotetext{
* Corresponding author.

E-mail address: simon.ruch@psy.unibe.ch (S. Ruch).

1 These authors contributed equally.

2 Senior author.
} 
during wakefulness (Breitenstein et al., 2005).

If the design of this vocabulary learning task invokes the episodic memory system, then conscious episodic learning in the waking state should profit from previous sleep-learning because both sleep- and awake-learning take place within the same memory system and potentially even within the same neural assemblies. Here, we tested for such a dual-learning benefit by giving participants five consecutive runs of conscious paired-associate vocabulary learning following their sleeplearning and implicit memory testing (Fig. 1B). Multiple runs of awake-learning (and retrieval) allowed delineating the steepness of awake-learning curves. We implemented three conditions of awakelearning (Fig. 1C): In the semantically congruent condition, we represented sleep-played foreign-words combined with synonyms to their sleep-played translation-words. We used synonyms rather than the sleep-played translation-words to avoid sole perceptual priming and increase chances of reactivating sleep-formed associations in episodic memory by demanding a flexible semantic conversion (guga-ship to guga-boat). Despite this semantic conversion, memory traces still build on each other because repeated learning in episodic memory relies on the meaning of items and strengthens memories when the material is congruent between learning runs (Höltje, Lubahn, \& Mecklinger, 2019; Staresina, Gray, \& Davachi, 2009; van Kesteren, Rijpkema, Ruiter, \& Fernández, 2010, 2013)). In the semantically incongruent condition, we expected the flattest learning curve because semantically conflicting translation-words were combined with sleep- and awake-presented foreign-words, which creates memory interference and lowers retrieval probability. In this condition, we combined the awakepresented foreign-words with synonyms to other sleep-played translation-words. In the baseline condition, we presented new pairs of foreignwords and translation-words to obtain an awake-learning curve that was uninfluenced by previous sleep-learning.

\section{Materials and methods}

\subsection{Participants}

We report the data of those 26 participants (age 19-32, mean \pm SD $=$ $22.96 \pm 3.41 ; 18$ (69\%) female), who were included in the behavioral part of the original study (Züst et al., 2019). Only those participants underwent the awake-learning procedure. The other group of 15 participants underwent neuroimaging during the implicit memory test without subsequent awake-learning.

The included participants were presented with vocabulary during sleep, took an implicit memory test following awakening, and engaged in conscious paired-associative learning over five runs. This study was approved by the local ethics committee "Kantonale Ethikkommission Bern".

\subsection{General procedure}

Participants arrived at the sleep laboratory at noon, after a night of partial sleep restriction. They gave written informed consent, were outfitted with electroencephalography electrodes and with in-ear headphones, took a hearing test, and then went to sleep for an afternoon nap. Throughout the entire nap, Brownian noise was presented via headphones at $65-74 \mathrm{~dB}(\mathrm{~A})$ SPL to reduce the salience of vocabulary presentations. Once the electroencephalogram (EEG) showed clearly visible slow-wave activity (SWS or stage 2 sleep "N2" at the transition to SWS), we initiated the acoustic presentation of word-pairs during sleep. Half of the sleep-played translation-words designated objects that are larger than a shoebox (e.g. ship) and half objects that are smaller than a shoebox (e.g. brush). Each word-pair was repeated four times in sequence with swapped positions (guga-ship, ship-guga, guga-ship, shipguga) to induce a flexible representation of the semantic word-word associations in episodic memory. Words were presented at a steady rhythm (onset interval between words of a pair: $1.075 \mathrm{~ms}$; onset interval between pairs: $4.3 \mathrm{~s}$ ) at an intensity at which participants had been able to identify $50 \%$ of number words "one" through "four" in the hearing test administered before sleep. Stimulus presentation was interrupted if participants showed an arousal or if they transitioned to lighter sleep stages. Stimulation was resumed once participants re-entered stable SWS. On average, we played 46.42 (SD 3.89) of the 48 word-pairs selected for sleep-learning during the $26.17 \mathrm{~min}$ that participants spent in SWS (SD $=9.00$; average nap duration $=95.17 \mathrm{~min}, \mathrm{SD}=$ 40.80 ; total sleep time $=68.27, \mathrm{SD}=31.45$ ). The total stimulation time was $13.31 \mathrm{~min}(\mathrm{SD}=1.12)$ per participant.

Following the sleep-presentation of the entire set of word-pairs, we woke up participants and gave them time to recover from sleep inertia.

A. Vocabulary Presentation during Sleep

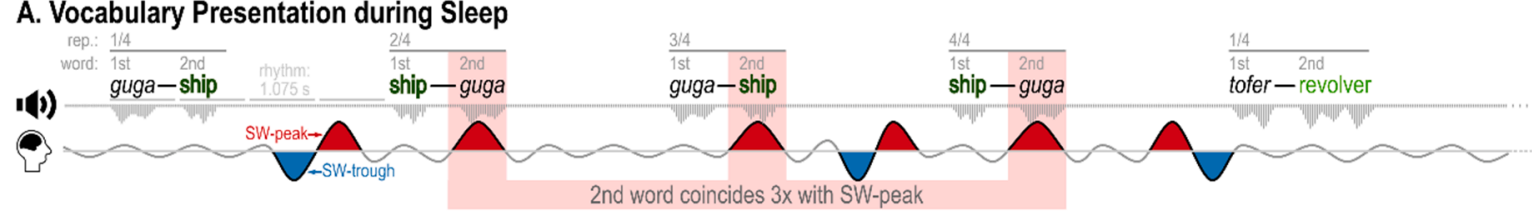

\section{B. Implicit Memory Test}

Size relative to a shoebox?

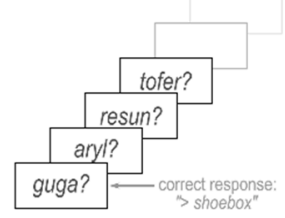

C. Awake-Learning \& Retrieval

RUN 1: Initial learning

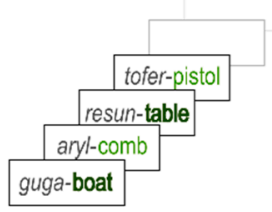

RUN 2-5: Retrieval \& relearning

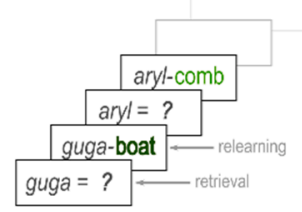

D. Awake-Learning Conditions

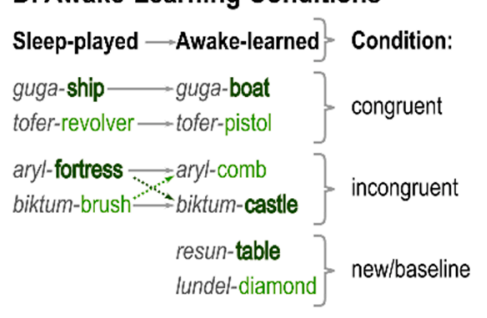

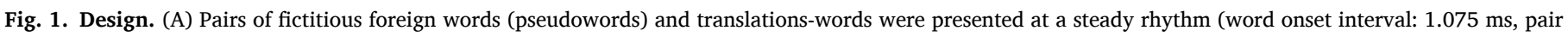

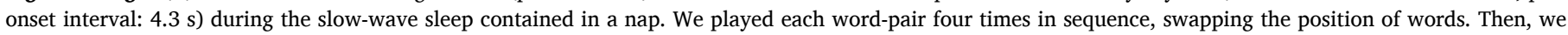

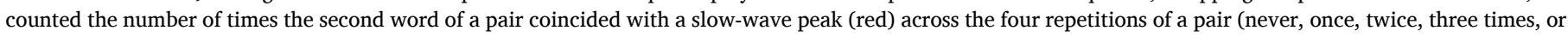

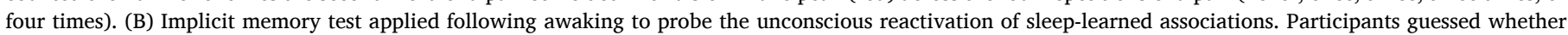

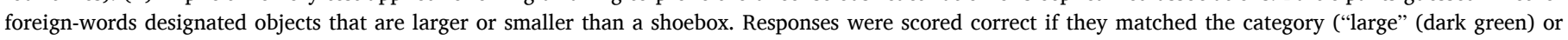

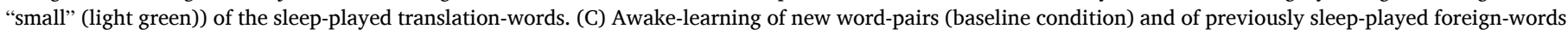

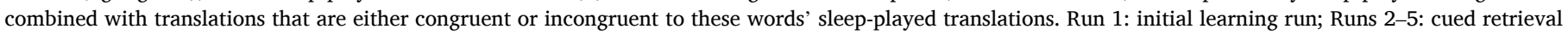

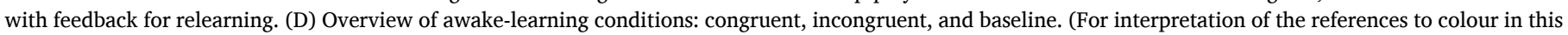
figure legend, the reader is referred to the web version of this article.) 
To prevent severe sleep inertia, we waited until participants entered a light sleep stage (N1 or N2) before waking them up. The average recovery time given following waking was $32.22 \mathrm{~min}(\mathrm{SD}=18.44 \mathrm{~min})$. As soon as participants felt ready to continue the experiment, they took an implicit memory test. The average time between the last sleep-played stimulus and the first stimulus in this test was $52.03 \mathrm{~min}(\mathrm{SD}=21.34$ ).

The implicit memory test required participants to guess via button press whether previously sleep-played foreign-words and new foreignwords designated objects that are larger or smaller than a shoebox (Fig. 1B). Responses were scored correct if they matched the category (large/small) of the sleep-played translation-words. Responses to new foreign-words were not scored.

Next, we asked participants to engage in five runs of pairedassociative vocabulary learning. Half of the sleep-played foreignwords were combined with synonyms to their sleep-played translations (congruent condition) and half were paired with synonyms to other sleep-played translation-words that came from the opposite semantic category (large/small) in order to induce a conflict (incongruent condition; Fig. 1C). New foreign-words that had not been played during sleep were presented with new translation-words to probe uninfluenced paired-associative learning (baseline condition). During the first of five learning runs, all word-pairs were presented for silent learning. In runs 2 to 5 , only the foreign-words were presented for participants to cuedrecall and state the translation words. Irrespective of the correctness of the response, the correct translation word was then presented for further paired-associative learning. Participants' verbal responses were recorded for later analysis. The experimenter gave the feedback when a participant had produced a response or - if no response was given - after $5 \mathrm{~s}$. The order of word-pairs was re-randomized for each learning run. Words were presented simultaneously visually on screen and acoustically via loudspeakers. At the end of the experiment, we asked participants whether they had heard anything while asleep. No participant reported having heard words or voices while asleep. Hence, sleep-played words were not perceived with conscious awareness.

\subsection{Stimulus material}

We generated two-syllabic pseudowords (e.g. guga) that we used as foreign-words of a fictional language. Using a fictional foreign language guaranteed that semantic paired-associative learning in this study was uninfluenced by multilingualism. Before pseudowords were assigned to experimental lists, five independent raters noted any associations that came to mind for each word. Pseudowords that were consistently associated with a specific real word or a specific word category (e.g. animals, or large objects) across raters were removed from the pool. The remaining pseudowords were compiled into three lists of 24 foreignwords each. Each list contained 24 synonym pairs that were used as translation-words for sleep-learning and ensuing awake-learning. Half of the synonym-pairs in each list denoted large objects (ship-boat), and half small objects (revolver-pistol). Lists were matched regarding word length, pronounceability, perceived concreteness of foreign-words, and lexical frequency of translation-words. Values for word pronounceability and concreteness were obtained from five independent raters. Lists were randomly assigned to the awake-learning conditions (congruent, incongruent, baseline) and counterbalanced over conditions. Within each list, pairs of foreign-words and translation-words were generated randomly for each participant. Translation-words selected for presentation during sleep (e.g. "ship" of "ship-boat") were never used as synonyms during awake-learning.

\subsection{Stimulus inclusion and exclusion}

Word-pairs intended for sleep-learning were excluded from the data analysis if any of the repetitions of a pair occurred in a sleep stage other than slow-wave rich N2 sleep or SWS, or during an arousal period. This led to an exclusion of 58 out of a total of 1207 word-pairs $(M=2.23$, SD
$=3.31$ pairs per participant; $\mathrm{M}=4.98 \%$, SD $=7.96 \%$ of pairs per participant).

\subsection{Electroencephalography}

Züst et al. (2019) had found that chances increased that sleeplearning yielded long-term memory traces if sleep slow-waves peaked between -275 and $25 \mathrm{~ms}$ of the onset of the second word in a pair. Because every word-pair was played four times in sequence, the second word could coincide once, twice, three times, four times, or never with a peak. Because awake-learning is bound to be influenced by the robustness of previous sleep-learning, we analyzed the steepness of the awakelearning curve by accounting for the number of slow-wave peaks that had coincided with the play of the second word of a pair. Here is how we determined whether the second word of a pair coincided with a slowwave peak: We first referenced the raw EEG signal (64 channels, 10-20 montage, $500 \mathrm{~Hz}$ sampling rate) to the global average and then extracted the average signal over frontal electrodes (F1, F2, Fz, FC1, FC2, FCz). Then, we low-pass filtered the resulting signal at $4 \mathrm{~Hz}$ and computed a time-frequency decomposition (Morlet wavelet transformations, two cycles in length) to extract the instantaneous phase of $0.8 \mathrm{~Hz}$ oscillations at $20 \mathrm{~ms}$ intervals. The second word of a pair was determined to coincide with a slow-wave peak, if the phase of $0.8 \mathrm{~Hz}$ oscillations was within $\pm 2.5 \%\left( \pm 9^{\circ}\right)$ of the peak phase in the time interval from $275 \mathrm{~ms}$ before to $25 \mathrm{~ms}$ following the onset of the second word. We determined the number of trials, in which the second word of a pair hit a slow-wave peak several times (2-4 times) and rarely (0/1 times) to contrast these two categories with respect to the later awakelearning success.

\subsection{Statistical analysis}

We performed logistic regressions at the single trial level to find out how retrieval success on the implicit memory test and retrieval success on the runs given for awake-learning would disperse with respect to the number of slow-wave peak-associated word presentations $(0 / 1$ times versus 2-4 times) and awake-learning condition (congruent/incongruent/baseline). The binary outcomes were guessing accuracy (correct/incorrect) on the implicit memory test and recall success (yes/no) on each awake-learning run. To assess the influence of successful implicit retrieval of sleep-learned associations on awake-learning, we performed additional analyses, in which we entered guessing accuracy on the implicit memory test as factor to predict recall success during awake-learning. We modeled random intercepts for participants and foreign-words (mixed effects regressions) when considering the awakelearning runs because both variables accounted significantly for variance in the awake-learning task. Analyses were performed in R (v4.0.2; $\mathrm{R}$ Core Team, 2020). We fitted binomial generalized linear models ("glm" function, R-package "stats") or mixed models ("glmer" function, R-package "lme4", v1.1-23; Bates, Mächler, Bolker, \& Walker, 2015) with a logit link.

We fitted full models that included all relevant predictors and all possible interaction terms. To assess the significance of each term, we performed type-II analyses of variance and reported Wald $\mathrm{X}_{\mathrm{W}}{ }^{2}$ statistics ("Anova" function, R-package "car", v3.0-9; Fox \& Weisberg, 2019). We further computed Bayes factors for each effect by analyzing how the fit of a Bayesian binary logistic (mixed) regression model improved when the effect of interest was added. Bayesian models were fitted with the Rpackage "brms" (v2.14.0) (Bürkner, 2017, 2018) (4 chains, 1000 warmup and 4000 sampling iterations) using a Bernoulli distribution (logit link) and default uninformed priors. We estimated the Bayes factors with the "bayes_factor" function of the "brms" package. For post-hoc analyses of the performance on the implicit memory test, we computed $\mathrm{X}^{2}$ tests. Corresponding Bayes factors were obtained using the "proportionBF" function (R-package "BayesFactor", v0.9.12-4.2; Morey, Rouder, Jamil, Urbanek, Forner, \& Ly, 2018). 


\section{Results}

Added over all 26 participants, a total of 1149 word-pairs was played during SWS. In a subtotal of 304 word-pairs, the second word of a pair hit a slow-wave peak twice, three or four times in the four repetitions of a word-pair. In a subtotal of 845 word-pairs, the second word of a pair never or only once hit a slow-wave peak in the four repetitions of a wordpair.

Participants' guessing accuracy on the implicit memory test was influenced by the number of times the second word of a sleep-played word-pair hit a slow-wave peak (effect of peaks $(0 / 1$ vs. $2-4): X_{W}{ }^{2}(1)$ $\left.=6.848, p=.009, B F_{10}=10.474\right)$. Accuracy exceeded the chance level of $50 \%$ (accuracy: $58.2 \%, X^{2}(1)=8.224$, $p_{\text {Bonferroni, } 2 \text { tests }}=0.008, B F_{10}=$ 8.064), if the second word of a pair had coincided with a slow-wave peak in at least two of the four repetitions ( $0 / 1$ peaks: accuracy $=49.6 \%$, $\mathrm{X}^{2}(1)=0.058, p_{\text {Bonferroni, } 2 \text { tests }}>0.999, B F_{10}=0.088$ ) (Fig. 2A) (Züst et al., 2019). Hence, repeated peak-associated stimulus presentation provided optimal conditions for sleep-learning.

Participants' cued-recall performance during awake-learning increased from run to run $\left(X_{W}^{2}(1)=713.989, p<.001, B F_{10}>10^{3}\right)$. Condition modulated recall performance (congruent/incongruent/ baseline; $X_{W}^{2}(2)=1.057, p=.589, B F_{10}=0.057$ ) only in interaction with the number of times the second word of a sleep-played pair hit a slow-wave peak (interaction: condition*peaks: $X_{W}^{2}(1)=3.947, p=$ $.047, B F_{10}=3.235$ ) (Fig. 2B). Awake-learning in the congruent condition was paradoxically diminished (rather than enhanced) when conditions for sleep-learning had been optimal (second word repeatedly hit peaks across repetitions) versus poor (second word hit peak once or never) $\left(X_{W}{ }^{2}(1)=6.676\right.$, $\left.p_{\text {Bonferroni, } 4 \text { tests }}=0.039, B F_{10}=10.493\right)$. Awakelearning in the congruent versus incongruent condition was diminished if sleep-learning conditions had been optimal $\left(X_{W}{ }^{2}(1)=6.330\right.$, $p_{\text {Bonferroni, }}$ 4 tests $\left.=0.048, B F_{10}=9.556\right)$, but not if sleep-learning conditions had been poor $\left(X_{W}^{2}(1)=0.132\right.$, $\left.p_{\text {Bonferroni, } 4 \text { tests }}>0.999, B F_{10}=0.265\right)$. Awake-learning in the incongruent condition was not modulated by the conditions during sleep-learning (peaks: $X_{W}^{2}(1)=0.745$, $p_{\text {Bonferroni, } 4 \text { tests }}$ $\left.>0.999, B F_{10}=0.515\right)$. Moreover, awake-learning in the congruent condition following optimal sleep-learning conditions was also diminished when compared to the awake-learning performance in the baseline condition, where word-pairs had not been played during sleep $\left(X_{W}^{2}(1)\right.$ $=6.606$, $\left.p_{\text {Bonferroni, } 4 \text { tests }}=0.041, B F_{10}=9.526\right)$. The retrieval performance in the congruent condition following optimal sleeplearning conditions dropped $>10 \%$ in the fifth run $(33.5 \%$ vs. $43.9 \%$ recalled associations). Neither recall performance in the incongruent condition nor recall performance in the congruent condition following poor sleep-learning conditions differed from the recall performance in the baseline condition (all $p_{\text {Bonferroni, } 4 \text { tests }}>0.999$, all $B F_{10}<0.350$ ).

A successful implicit retrieval of sleep-played word-pairs tended to expedite the awake-learning of both congruent and incongruent wordpairs, albeit not above the awake-learning performance observed in the baseline condition. In fact, both implicit memory (correct/incorrect responses given on the shoebox task that constituted the implicit memory test) and the awake-learning condition (congruent/incongruent) modulated retrieval performance independently of each other and interacted with the number of times the second word of a sleepplayed word-pair had hit a slow-wave peak (condition * peaks: $X_{W}^{2}(1)=4.619, p=0.032, B F_{10}=4.881$; implicit memory * peaks: $\left.X_{W}^{2}(1)=8.976, p=0.003, B F_{10}=44.001\right)$. If sleep-learning conditions had been optimal (2-4 peaks), awake-learning was diminished in the congruent versus the incongruent condition (main effect of condition: $X_{W}{ }^{2}(1)=6.117, p_{\text {Bonferroni, } 2 \text { tests }}=0.027, B F_{10}=10.754$ ), but was expedited somewhat following a successful versus unsuccessful implicit retrieval (main effect of implicit memory: $X_{W}^{2}(1)=9.985$, $p_{\text {Bonferroni, } 2}$ tests $=0.003, B F_{10}=129.215$ ) (Fig. 3). Importantly, the influence of implicit memory performance on awake-learning was not significantly modulated by condition (condition * implicit memory: $X_{W}^{2}(1)=0.498$, $p_{\text {Bonferroni, } 2 \text { tests }}=0.961, B F_{10}=1.025$ ), although the Bayes Factor was inconclusive regarding the presence or absence of an interaction. Neither condition nor implicit memory significantly modulated awakelearning following poor sleep-learning conditions (all $p_{\text {Bonferroni, } 2 \text { tests }}$ $>0.180$, all $B F_{10}<1.002$ ). If sleep-learning conditions had been optimal and the choice on the implicit retrieval test was incorrect, awakelearning in the congruent condition was significantly diminished compared to awake-learning in the baseline condition $\left(X_{W}{ }^{2}(1)=9.173\right.$, $p_{\text {Bonferroni, } 8 \text { tests }}=0.020, B F_{10}=62.048$ ). If sleep-learning conditions had been optimal and the choice on the implicit retrieval test was correct, awake-learning in the congruent condition was not significantly different from the awake-learning in the baseline condition $\left(X_{W}{ }^{2}(1)=\right.$ 0.885 , $\left.p_{\text {Bonferroni, } 8 \text { tests }}>0.999, B F_{10}=0.657\right)$. Hence, a successful implicit retrieval performance tended to compensate for the negative effect of sleep-learning on awake-learning. No other condition significantly
A. Implicit Memory

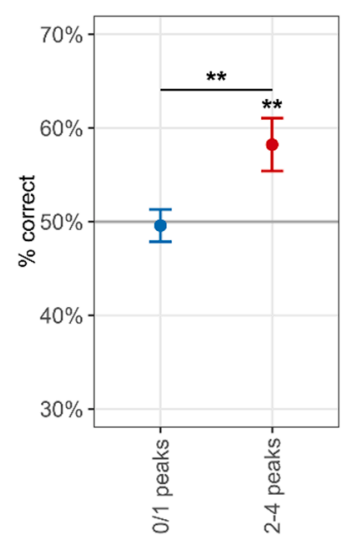

B. Awake-Learning \& Retrieval
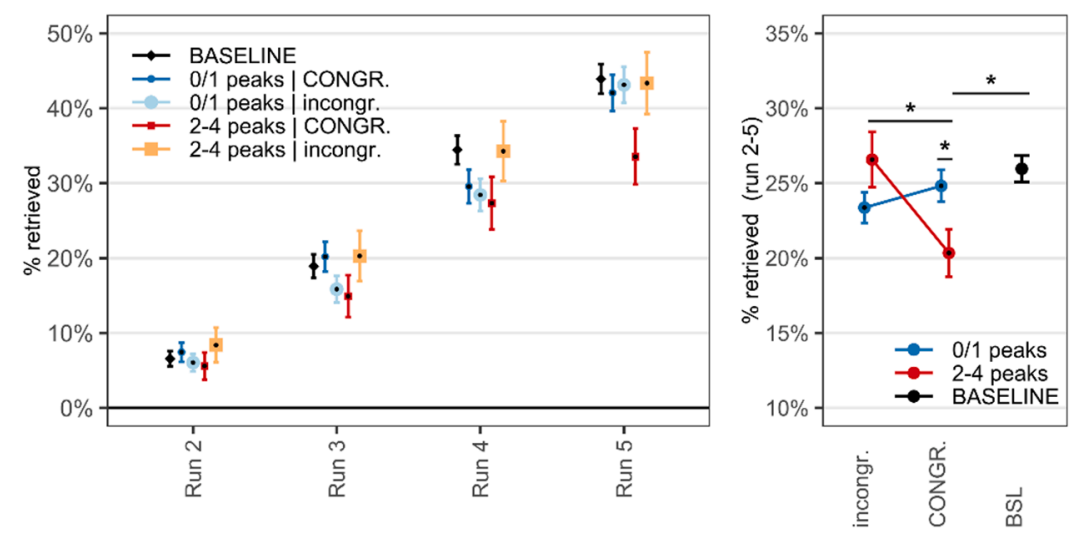

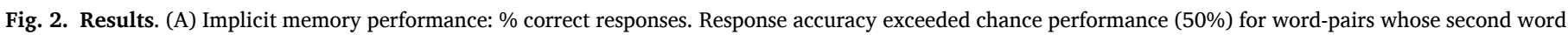

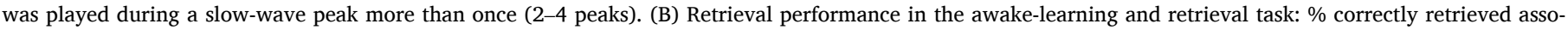

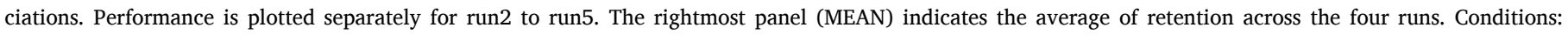

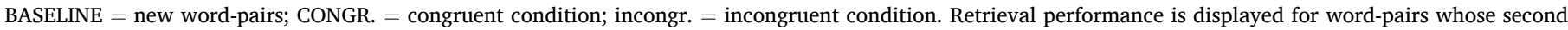

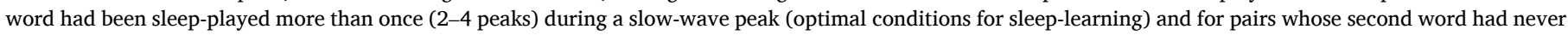

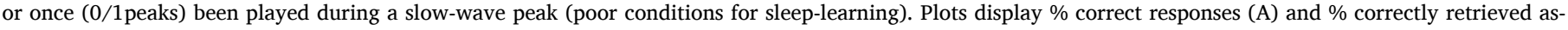
sociations (B) at the single-trial level. Error bars indicate binomial standard errors. **p $<.01$, *p $<.05$. 


\section{Awake-Learning \& Retrieval by Implicit Memory Performance}
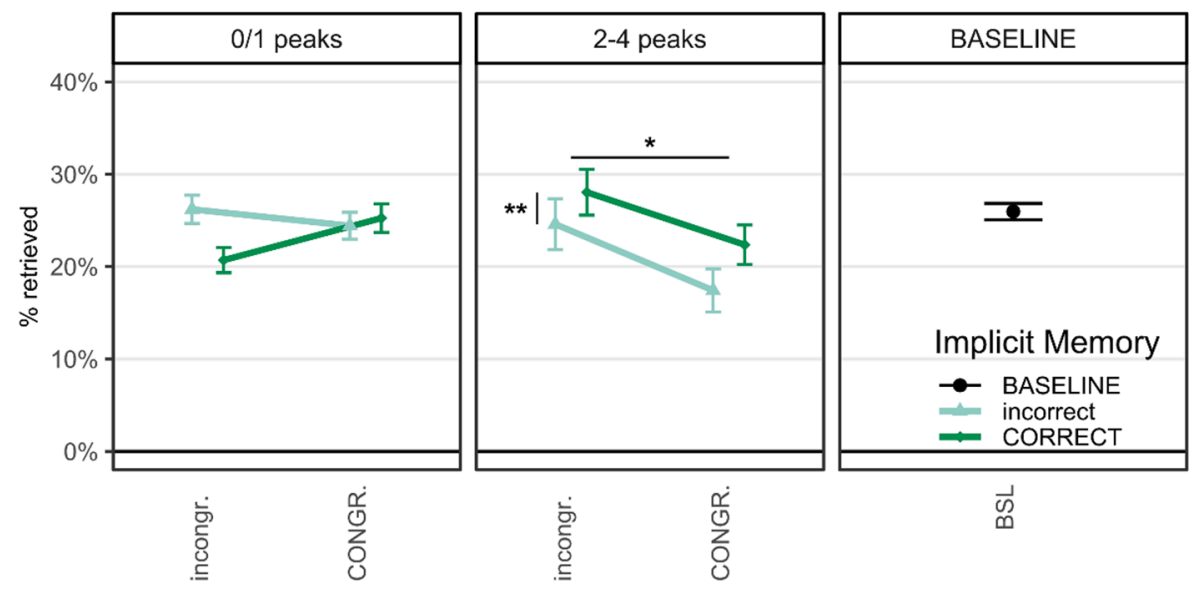

deviated from baseline (all $p_{\text {Bonferroni, } 8 \text { tests }}>0.999$, all $B F_{10}<0.8$ ).

\section{Discussion}

We explored whether sleep-learning expedites awake-learning of semantically congruent associations. Paradoxically, sleep-learning diminished rather than promoted awake-learning of semantically congruent associations but left the awake-learning of incongruent associations uninfluenced. Although the conscious encoding of information that is congruent/incongruent to stored information is known to promote/diminish memory formation (Höltje et al., 2019; van Kesteren et al., 2010), the present switchover from the sleeping to the waking state has apparently altered this interaction. However, this altered interaction is not unprecedented: Andrillon et al. (2017) gave their participants a perceptual learning task during slow-wave sleep and again following awakening. Participants' awake ability to learn to detect repeating noise patterns in the stream of random noise was impaired if the same noise patterns had been presented during SWS, but not if the patterns were new or had been presented during REM sleep or light nonREM sleep (Andrillon et al., 2017). What was the mechanism that lowered awake-learning following sleep-learning? According to Andrillon et al. (2017), stimulus presentations during SWS were associated with a stronger-than-normal synaptic downscaling in neurons that are activated by the sleep-played stimuli. Synaptic downscaling renews the learning capacity of the brain by eliminating unused and irrelevant synapses (Tononi \& Cirelli, 2014). Andrillon et al. (2017) surmised that an exaggerated downscaling may have impaired their participants' ability to process and store the sleep-played information following waking. The present data are incompatible with an exaggerated downscaling in stimulus-specific networks because participants had learned the sleep-played vocabulary and retrieved it on the implicit memory test administered following awakening. Learning mostly requires synaptic potentiation (Takeuchi, Duszkiewicz, \& Morris, 2014), which is probably why sleep-learning in the present study was successful only during peaks of slow-waves when synaptic potentiation is possible (Andrillon \& Kouider, 2020; Destexhe et al., 2007). We therefore speculate that semantic associative learning may have interfered with the normal synaptic downscaling in those cortico-hippocampal neurons that were activated during sleep-learning and - due to their semantic specialization - were again activated during awake-learning of the same/similar semantic associations. Indeed, neurons can be spared from downscaling if they are highly active during sleep (Gulati, Guo, Ramanathan, Bodepudi, \& Ganguly, 2017; Tononi \& Cirelli, 2020). If neurons that were dually involved in sleep-learning and awake-learning had escaped
Fig. 3. Effect of implicit memory performance on awake-learning and retrieval. Retrieval performance on the awake-learning and retrieval task: $\%$ correctly retrieved associations, averaged across the four retrieval runs. Performance is plotted separately for Conditions (BSL/BASELINE = new word-pairs; CONGR. = congruent condition; incongr. = incongruent condition), sleep-learning conditions (2-4 peaks: optimal conditions; 0/1 peaks: poor conditions) and implicit memory performance (CORRECT vs. incorrect classification of foreign words on the implicit memory test, i.e., the shoebox task). Both condition and implicit memory performance modulated retrieval performance independently of each other following optimal sleep-learning conditions. Error bars indicate binomial standard errors. **p $<.01,{ }^{*} \mathrm{p}<.05$. downscaling, then their learning capacity is deemed insufficient for normal awake-learning. The fact that awake-learning of new semantic associations in the incongruent condition proceeded undisturbed and at a normal level further suggests that the escape from downscaling has only then negative consequences when neurons are dually recruited for sleep- and awake-learning due to their semantic specialization. If this interpretation is correct, a recovery nap between sleep-learning and awake-learning might restore synaptic homeostasis (Mander, Santhanam, Saletin, \& Walker, 2011) and learning capacity, which might then leave awake-learning unimpaired or even enhance it.

The proposed downscaling mechanism, by which sleep-formed memories interfere with wake-learning, appears to be unique to learning during sleep and does not seem to occur during wakefulness. Indeed, repeated learning runs given for awake-learning improve episodic memory (Tabibian et al., 2019) and awake-formed prior knowledge facilitates the subsequent awake-learning of related information (Greve, Cooper, Tibon, \& Henson, 2019). Also, in the present study, there was an increase in memory performance with each awakelearning run (Fig. 2B). Of note, even awake-formed unconscious memories built from subliminally presented images facilitated the subsequent conscious, supraliminal learning of the same information (Ruch, Herbert, \& Henke, 2017). So why do repeated learning runs invariably improve rather than impair episodic memory, when the initial learning occurs at wake rather than sleep? Because distinct plasticity mechanisms are at play during wake versus sleep (Tononi \& Cirelli, 2014). The awake brain is prepared for synaptic potentiation (Vyazovskiy, Cirelli, PfisterGenskow, Faraguna, \& Tononi, 2008). Episodic learning at wake induces a strong and specific synaptic potentiation that benefits the subsequent re-encoding of the same or similar information. If the engaged synapses are saturated, or if synaptic saturation is generally high due to prolonged wakefulness (Kurinec, Whitney, Hinson, Hansen, \& Van Dongen, 2021; Ruch, Valiadis, \& Gharabaghi, 2021), then even at wake additional learning runs will lead to diminishing performance gains. Nevertheless, repeated runs of awake-learning will always improve rather than impair memory performance. Yet, once asleep, the brain is no longer set for synaptic potentiation. At the contrary, sleep sets the brain for synaptic downscaling (Tononi \& Cirelli, 2014; Vyazovskiy et al., 2008) as mentioned in the previous paragraph. Although some potentiation and learning are still possible at certain times during sleep (Brzosko, Mierau, \& Paulsen, 2019; González-Rueda, Pedrosa, Feord, Clopath, \& Paulsen, 2018), sleep-learning likely yields weak memories both in terms of the number of synapses recruited for learning and in terms of the amount of synaptic potentiation. Not only does sleep-learning interfere with the natural sleep-associated process of synaptic renormalization in those 
neurons that are recruited for sleep-learning, but the escape from renormalization also prevents these same neurons from getting rid of unnecessary information that they had acquired during the previous wake time. Later, during the conscious awake-learning of information, which is semantically similar to the sleep-learned information, the neurons recruited for sleep-learning will be recruited again due to their semantic specialization. But these neurons are saturated and they still hold the irrelevant information that they had acquired during the previous wake period because they escaped synaptic renormalization during sleep. Therefore, these neurons are unable to provide an awakelearning efficiency comparable to other neurons that had not been recruited for sleep-learning. Metaphorically speaking, sleep-learning is thus like writing on a chalkboard while someone else is trying to clean the board (=down-scaling) to remove an older drawing: writing interferes with the cleaning process and leaves only a barely legible trace. Re-tracing the newly written text later on (=awake relearning) does not make it more legible because parts of the old drawing are still there, whereas writing new text on a cleaned area on the chalkboard (=awake learning of new information) provides clearly legible text.

Interestingly, the successful versus unsuccessful implicit retrieval of sleep-played associations enhanced the subsequent awake-learning slightly in both the congruent and incongruent condition. This enhancement of awake-learning in the congruent and incongruent condition was mild with the respective retrieval performance not rising above the retrieval performance measured in the baseline condition. Because a successful versus unsuccessful implicit retrieval of sleepplayed associations enhanced awake-learning in both the congruent and incongruent conditions, the enhancing effect must originate in an increased perceptual fluency of the foreign words alone and cannot result from the sleep-formed semantic associations between foreign words and translation words. The increased perceptual fluency may have aided the awake-learning of semantically congruent and semantically incongruent associations because this fluency facilitated the processing of the foreign words and as a consequence their association with the translation words. This mechanism of interaction is likely because a large perceptual fluency (Sanchez \& Naylor, 2018) and familiarity (Dennis, Turney, Webb, \& Overman, 2015; Kilb \& Naveh-Benjamin, 2011) improve relational encoding and retrieval. Even wordpresentations during sleep influenced the processing of these same words during subsequent wakefulness (Andrillon \& Kouider, 2016; Ruch et al., 2014).

As in our previous work (Züst et al., 2019), we posit that vocabulary presentations during sleep had triggered a hippocampus-mediated rapid semantic-associative encoding process that provided for flexible representations of combinations of foreign words and translation words in episodic memory. We now discuss a potential alternative mechanism of sleep-learning that involves a mere phonological/perceptual encoding process. If sleep-learning were perceptual, the hippocampal activity we had observed (Züst et al., 2019) at wake implicit memory testing for correct versus incorrect retrieval responses might have occurred because participants used the reactivated phonological memory to decide about the physical size of the object that is referred to by the foreign words presented at test. This may have activated semantic networks and the hippocampus. Because the sleep-formed associations were of phonological/perceptual nature according to this mechanism, sleep-learning likely resulted from a purely neocortical mechanism (see e.g. Breitenstein et al., 2005). We believe that this phonological/perceptual sleeplearning account is unlikely for the following reasons. First, Andrillon et al. (2017) reported that auditory perceptual learning during slowwave sleep was not possible or led to suppressive memory traces that could not be retrieved following awakening. However, the sleep-formed associations were retrieved following awaking in our study (Züst et al., 2019). Second, a hippocampal rather than neocortical learning mechanism is suggested by the speed of the sleep-generated associative learning process and by the compositional representation of the sleepformed associations: Each pair of a foreign word and a translation was presented only four times requiring a rapid encoding. The implicit retrieval task required a cued recall because only half of the learned information (the foreign word alone) was presented at test. The cued recall format necessitates a compositional representation of the formed word-word associations. Only the hippocampus is capable of forming arbitrary word-word associations rapidly and storing the associations in a compositional format. The neocortex forms new arbitrary associations only slowly over dozens of learning trials and provides for fused (rather than compositional) word-word representations in memory (Cohen \& Eichenbaum, 1993; Henke, 2010; O'Reilly, Bhattacharyya, Howard, \& Ketz, 2014). Third, a semantic rather than perceptual sleep-learning mechanism is suggested by the fact that the actual percept of a word pair changed with every repetition because we switched the order of the two words between their presentations (i.e., guga-ship, ship-guga, gugaship, ship-guga). Furthermore, we presented the word-pairs embedded in constantly playing random noise. This perceptual variability and noise renders a mere phonological learning mechanism unlikely (Banai \& Lavie, 2020). Fourth, much evidence suggests that the necessary preconditions for hippocampus-mediated semantic paired-associative learning are fulfilled during SWS. Indeed, humans are able to process the semantic content of verbal messages during sleep (Strauss \& Dehaene, 2019). Sensory stimulation during sleep was found to activate the hippocampus and to trigger memory reactivation and memory consolidation both in animals (Bendor \& Wilson, 2012) and in humans (Rasch, Büchel, Gais, \& Born, 2007). Fifth, animal studies using intracranial brain stimulation suggested that the hippocampus maintains its ability to form new memories during slow-wave sleep (de Lavilléon, Lacroix, Rondi-Reig, \& Benchenane, 2015). Sixth, we have measured the brain activity with fMRI in five persons while they learned pairs of foreign words and translation words during slow-wave sleep and found the hippocampus activated (besides language areas) in all persons (unpublished follow-up study). Because the learning material was similar to the material given for sleep-learning in the current study, we assume that the learning-related neural network in the current study was similar to the network observed in these five persons.

The explanatory power of this study is limited by the fact that we performed neither functional neuroimaging nor intracerebral recordings during sleep-learning and awake-learning. Hence, we cannot elucidate the precise neuronal mechanisms underlying the observed sleeplearning and awake-learning effects. We can only assume that sleeplearning corrupted the ongoing synaptic downscaling in those neural assemblies that mediated semantic relational learning during sleep and during subsequent wakefulness in the congruent condition. This assumption builds on the notion that SWS plays a critical role in synaptic downscaling. While there is some evidence that REM sleep might also contribute to synaptic downscaling (Grosmark, Mizuseki, Pastalkova, Diba, \& Buzsáki, 2012) and that SWS may even benefit a synaptic upscaling (Chauvette, Seigneur, \& Timofeev, 2012; Kim, Gulati, \& Ganguly, 2019; see Klinzing, Niethard, \& Born, 2019 for an in-depth discussion), the bulk of evidence associates SWS with synaptic downscaling (for a discussion see Cirelli \& Tononi, 2021; Niethard \& Born, 2019).

In sum, our findings reveal that associative learning during sleep is possible and comes at the cost of an interference with subsequent awake associative learning of the same or semantically similar item-pairs. Fortunately, the negative impact of sleep-learning on awake-learning is small. Nevertheless, the present findings highlight the importance of a quiet sleep environment. Although auditory stimulation during sleep can be used to enhance slow-wave sleep (Wunderlin et al., 2021) and to trigger memory reactivation and memory consolidation during sleep (Hu, Cheng, Chiu, \& Paller, 2020), auditory stimulation can also impair sleep quality (Göldi \& Rasch, 2019) and sleep's recuperative function. Our findings further suggest that meaningful sounds or verbal messages played during sleep may trigger learning processes that have potentially unwanted effects on awake behavior. Hence, caution is advised in using auditory stimulation for targeting memory reactivation and boosting 
memory consolidation during sleep (Hu et al., 2020).

\section{CRediT authorship contribution statement}

Simon Ruch: Conceptualization, Methodology, Investigation, Formal analysis, Resources, Writing - original draft, Writing - review \& editing, Visualization. Marc Alain Züst: Conceptualization, Methodology, Investigation, Resources, Writing - review \& editing, Funding acquisition. Katharina Henke: Conceptualization, Methodology, Resources, Writing - review \& editing, Supervision, Project administration, Funding acquisition.

\section{Declaration of Competing Interest}

The authors declare that they have no known competing financial interests or personal relationships that could have appeared to influence the work reported in this paper.

\section{Acknowledgments}

This work was supported by the Interfaculty Research Cooperation grant "Decoding Sleep: From Neurons to Health and Mind" (to K.H.) from the University of Bern and the Swiss National Science Foundation (SNSF) grant P0BEP1_148941-1 (to M.A.Z.).

\section{Data availability}

The data and analyses relevant for this study are available on https:// doi.org/10.17605/OSF.IO/ZSG5F.

\section{References}

Andrillon, T., \& Kouider, S. (2016). Implicit memory for words heard during sleep. Neuroscience of Consciousness, 2016(1), niw014. https://doi.org/10.1093/nc/niw014

Andrillon, T., \& Kouider, S. (2020). The vigilant sleeper: Neural mechanisms of sensory (de)coupling during sleep. Current Opinion in Physiology, 15, 47-59. https://doi.org/ 10.1016/j.cophys.2019.12.002

Andrillon, T., Pressnitzer, D., Léger, D., \& Kouider, S. (2017). Formation and suppression of acoustic memories during human sleep. Nature Communications, 8(1), 179. https://doi.org/10.1038/s41467-017-00071-z

Arzi, A., Holtzman, Y., Samnon, P., Eshel, N., Harel, E., \& Sobel, N. (2014). Olfactory aversive conditioning during sleep reduces cigarette-smoking behavior. The Journal of Neuroscience, 34(46), 15382-15393. https://doi.org/10.1523/JNEUROSCI.229114.2014

Arzi, A., Shedlesky, L., Ben-Shaul, M., Nasser, K., Oksenberg, A., Hairston, I. S., \& Sobel, N. (2012). Humans can learn new information during sleep. Nature Neuroscience, 15, 1460-1465. https://doi.org/10.1038/nn.3193

Banai, K., \& Lavie, L. (2020). Rapid perceptual learning and individual differences in speech perception: The good, the bad, and the sad. Auditory Perception \& Cognition, 3 (4), 201-211. https://doi.org/10.1080/25742442.2021.1909400

Bates, D., Mächler, M., Bolker, B., \& Walker, S. (2015). Fitting linear mixed-effects models using lme4. Journal of Statistical Software, 67(1), 1-48. https://doi.org/ 10.18637/jss.v067.i01

Bendor, D., \& Wilson, M. A. (2012). Biasing the content of hippocampal replay during sleep. Nature Neuroscience. https://doi.org/10.1038/nn.3203

Breitenstein, C., Jansen, A., Deppe, M., Foerster, A.-F., Sommer, J., Wolbers, T., \& Knecht, S. (2005). Hippocampus activity differentiates good from poor learners of a novel lexicon. NeuroImage, 25(3), 958-968. https://doi.org/10.1016/j. neuroimage.2004.12.019

Brzosko, Z., Mierau, S. B., \& Paulsen, O. (2019). Neuromodulation of spike-timingdependent plasticity: Past, present, and future. Neuron, 103(4), 563-581. https:// doi.org/10.1016/j.neuron.2019.05.041

Bürkner, P.-C. (2017). brms: An R package for Bayesian multilevel models using Stan. Journal of Statistical Software, 80(1), 1-28. https://doi.org/10.18637/jss.v080.i01

Bürkner, P.-C. (2018). Advanced Bayesian multilevel modeling with the R package brms. The R Journal, 10(1), 395-411.

Chauvette, S., Seigneur, J., \& Timofeev, I. (2012). Sleep oscillations in the thalamocortical system induce long-term neuronal plasticity. Neuron, 75(6), 1105-1113. https://doi.org/10.1016/j.neuron.2012.08.034

Cirelli, C., \& Tononi, G. (2021). The why and how of sleep-dependent synaptic downselection. Seminars in Cell \& Developmental Biology. https://doi.org/10.1016/j. semcdb.2021.02.007

Cohen, N. J., \& Eichenbaum, H. (1993). Memory, amnesia, and the hippocampal system. MIT Press.
Cox, R., Korjoukov, I., de Boer, M., \& Talamini, L. M. (2014). Sound asleep: Processing and retention of slow oscillation phase-targeted stimuli. PLOS ONE, 9(7), Article e101567. https://doi.org/10.1371/journal.pone.0101567

de Lavilléon, G., Lacroix, M. M., Rondi-Reig, L., \& Benchenane, K. (2015). Explicit memory creation during sleep demonstrates a causal role of place cells in navigation. Nature Neuroscience, 18, 493-495. https://doi.org/10.1038/nn.3970

Dennis, N. A., Turney, I. C., Webb, C. E., \& Overman, A. A. (2015). The effects of item familiarity on the neural correlates of successful associative memory encoding. Cognitive, Affective, \& Behavioral Neuroscience, 15(4), 889-900. https://doi.org/ 10.3758/s13415-015-0359-2

Destexhe, A., Hughes, S. W., Rudolph, M., \& Crunelli, V. (2007). Are corticothalamic 'up' states fragments of wakefulness? Trends in Neurosciences, 30(7), 334-342. https:// doi.org/10.1016/j.tins.2007.04.006

Fox, J., \& Weisberg, S. (2019). An R companion to applied regression (3rd ed.). SAGE Publications.

Göldi, M., \& Rasch, B. (2019). Effects of targeted memory reactivation during sleep at home depend on sleep disturbances and habituation. Npj Science of Learning, 4(1), 5. https://doi.org/10.1038/s41539-019-0044-2

González-Rueda, A., Pedrosa, V., Feord, R. C., Clopath, C., \& Paulsen, O. (2018). Activitydependent downscaling of subthreshold synaptic inputs during slow-wave-sleep-like activity in vivo. Neuron, 97(6), 1244-1252.e5. https://doi.org/10.1016/j. neuron.2018.01.047

Greve, A., Cooper, E., Tibon, R., \& Henson, R. N. (2019). Knowledge is power: Prior knowledge aids memory for both congruent and incongruent events, but in different ways. Journal of Experimental Psychology. General, 148(2), 325-341. https://doi.org/ 10.1037/xge0000498

Grosmark, A. D., Mizuseki, K., Pastalkova, E., Diba, K., \& Buzsáki, G. (2012). REM sleep reorganizes hippocampal excitability. Neuron, 75(6), 1001-1007. https://doi.org/ 10.1016/j.neuron.2012.08.015

Gulati, T., Guo, L., Ramanathan, D. S., Bodepudi, A., \& Ganguly, K. (2017). Neural reactivations during sleep determine network credit assignment. Nature Neuroscience, 20, 1277-1284. https://doi.org/10.1038/nn.4601

Henke, K. (2010). A model for memory systems based on processing modes rather than consciousness. Nature Reviews Neuroscience, 11(7), 523-532. https://doi.org/ $10.1038 / \mathrm{nrn} 2850$

Höltje, G., Lubahn, B., \& Mecklinger, A. (2019). The congruent, the incongruent, and the unexpected: Event-related potentials unveil the processes involved in schematic encoding. Neuropsychologia, 131, 285-293. https://doi.org/10.1016/j. neuropsychologia.2019.05.013

Hu, X., Cheng, L. Y., Chiu, M. H., \& Paller, K. A. (2020). Promoting memory consolidation during sleep: A meta-analysis of targeted memory reactivation. Psychological Bulletin, 146(3), 218-244. https://doi.org/10.1037/bul0000223

Kilb, A., \& Naveh-Benjamin, M. (2011). The effects of pure pair repetition on younger and older adults' associative memory. Journal of Experimental Psychology. Learning Memory, and Cognition, 37(3), 706-719. https://doi.org/10.1037/a0022525

Kim, J., Gulati, T., \& Ganguly, K. (2019). Competing roles of slow oscillations and delta waves in memory consolidation versus forgetting. Cell, 179(2), 514-526.e13. https://doi.org/10.1016/j.cell.2019.08.040

Klinzing, J. G., Niethard, N., \& Born, J. (2019). Mechanisms of systems memory consolidation during sleep. Nature Neuroscience, 22(10), 1598-1610. https://doi. org/10.1038/s41593-019-0467-3

Kurinec, C. A., Whitney, P., Hinson, J. M., Hansen, D. A., \& Van Dongen, H. P. A. (2021). Sleep deprivation impairs binding of information with its context. Sleep, 44(8), zsab113. https://doi.org/10.1093/sleep/zsab113

Mander, B. A., Santhanam, S., Saletin, J. M., \& Walker, M. P. (2011). Wake deterioration and sleep restoration of human learning. Current Biology, 21(5), 183-184. https:// doi.org/10.1016/j.cub.2011.01.019

Morey, R. D., Rouder, J. N., Jamil, T., Urbanek, S., Forner, K., \& Ly, A. (2018). BayesFactor: Computation of Bayes factors for common designs (0.9.12-4.2) [Computer software]. https://CRAN.R-project.org/package=BayesFactor.

Niethard, N., \& Born, J. (2019). Back to baseline: Sleep recalibrates synapses. Nature Neuroscience, 1. https://doi.org/10.1038/s41593-018-0327-6

O'Reilly, R. C., Bhattacharyya, R., Howard, M. D., \& Ketz, N. (2014). Complementary learning systems. Cognitive Science, 38(6), 1229-1248. https://doi.org/10.1111/ j.1551-6709.2011.01214.x

R Core Team (2020). R: A language and environment for statistical computing (4.0.2) [Computer software]. https://www.R-project.org/.

Rasch, B., Büchel, C., Gais, S., \& Born, J. (2007). Odor cues during slow-wave sleep prompt declarative memory consolidation. Science, 315(5817), 1426-1429. https:// doi.org/10.1126/science.1138581

Ruch, S., \& Henke, K. (2020). Learning during sleep: A dream comes true? Trends in Cognitive Sciences, 24(3), 170-172. https://doi.org/10.1016/j.tics.2019.12.007

Ruch, S., Herbert, E., \& Henke, K. (2017). Subliminally and supraliminally acquired longterm memories jointly bias delayed decisions. Frontiers in Psychology, 8, 1542. https://doi.org/10.3389/fpsyg.2017.01542

Ruch, S., Koenig, T., Mathis, J., Roth, C., \& Henke, K. (2014). Word encoding during sleep is suggested by correlations between word-evoked up-states and post-sleep semantic priming. Frontiers in Psychology, 5, 1319. https://doi.org/10.3389/ fpsyg. 2014.01319

Ruch, S., Valiadis, M., \& Gharabaghi, A. (2021). Sleep to learn. Sleep, 44(8), zsab160. https://doi.org/10.1093/sleep/zsab160

Sanchez, C. A., \& Naylor, J. S. (2018). Disfluent presentations lead to the creation of more false memories. PLOS ONE, 13(1), Article e0191735. https://doi.org/10.1371/ journal.pone.0191735

Schabus, M., Dang-Vu, T. T., Heib, D. P. J., Boly, M., Desseilles, M., Vandewalle, G., ... Maquet, P. (2012). The fate of incoming stimuli during NREM sleep is determined by 
spindles and the phase of the slow oscillation. Frontiers in Sleep and Chronobiology, 3, 40. https://doi.org/10.3389/fneur.2012.00040

Sirota, A., \& Buzsáki, G. (2005). Interaction between neocortical and hippocampal networks via slow oscillations. Thalamus \& Related Systems, 3(4), 245-259. https:// doi.org/10.1017/S1472928807000258

Staresina, B. P., Gray, J. C., \& Davachi, L. (2009). Event congruency enhances episodic memory encoding through semantic elaboration and relational binding. Cerebral Cortex, 19(5), 1198-1207. https://doi.org/10.1093/cercor/bhn165

Strauss, M., \& Dehaene, S. (2019). Detection of arithmetic violations during sleep. Sleep, 42(3), 1-12. https://doi.org/10.1093/sleep/zsy232

Tabibian, B., Upadhyay, U., De, A., Zarezade, A., Schölkopf, B., \& Gomez-Rodriguez, M. (2019). Enhancing human learning via spaced repetition optimization. Proceedings of the National Academy of Sciences, 116(10), 3988-3993. https://doi.org/10.1073/ pnas. 1815156116

Takeuchi, T., Duszkiewicz, A. J., \& Morris, R. G. M. (2014). The synaptic plasticity and memory hypothesis: Encoding, storage and persistence. Philosophical Transactions of the Royal Society B: Biological Sciences, 369(1633), 20130288. https://doi.org/ $10.1098 /$ rstb.2013.0288

Tononi, G., \& Cirelli, C. (2014). Sleep and the price of plasticity: From synaptic and cellular homeostasis to memory consolidation and integration. Neuron, 81(1), 12-34. https://doi.org/10.1016/j.neuron.2013.12.025
Tononi, G., \& Cirelli, C. (2020). Sleep and synaptic down-selection. European Journal of Neuroscience, 51(1), 413-421. https://doi.org/10.1111/ejn.14335

van Kesteren, M. T. R., Beul, S. F., Takashima, A., Henson, R. N., Ruiter, D. J., \& Fernández, G. (2013). Differential roles for medial prefrontal and medial temporal cortices in schema-dependent encoding: From congruent to incongruent. Neuropsychologia, 51(12), 2352-2359. https://doi.org/10.1016/j. neuropsychologia.2013.05.027

van Kesteren, M. T. R., Rijpkema, M., Ruiter, D. J., \& Fernández, G. (2010). Retrieval of associative information congruent with prior knowledge is related to increased medial prefrontal activity and connectivity. The Journal of Neuroscience, 30(47), 15888-15894. https://doi.org/10.1523/JNEUROSCI.2674-10.2010

Vyazovskiy, V. V., Cirelli, C., Pfister-Genskow, M., Faraguna, U., \& Tononi, G. (2008). Molecular and electrophysiological evidence for net synaptic potentiation in wake and depression in sleep. Nature Neuroscience, 11(2), 200-208. https://doi.org/ 10.1038/nn2035

Wunderlin, M., Züst, M. A., Hertenstein, E., Fehér, K. D., Schneider, C. L., Klöppel, S., \& Nissen, C. (2021). Modulating overnight memory consolidation by acoustic stimulation during slow wave sleep - a systematic review and meta-analysis. Sleep, 44(7), zsaa296. https://doi.org/10.1093/sleep/zsaa296

Züst, M. A., Ruch, S., Wiest, R., \& Henke, K. (2019). Implicit vocabulary learning during sleep is bound to slow-wave peaks. Current Biology, 29(4), 541-553. https://doi.org/ 10.1016/j.cub.2018.12.038 